\title{
MAXIMAL RESIDUE DIFFERENCE SETS MODULO $p$
}

\author{
DUNCAN A. BUELL AND KENNETH S. WILLIAMS ${ }^{1}$
}

\begin{abstract}
Let $p \equiv 1(\bmod 4)$ be a prime. A residue difference set modulo $p$ is a set $S=\left\{a_{i}\right\}$ of integers $a_{i}$ such that $\left(\frac{a_{i}}{p}\right)=+1$ and $\left(\frac{a_{i}-a_{j}}{p}\right)=+1$ for all $i$ and $j$ with $i \neq j$, where $\left(\frac{n}{p}\right)$ is the Legendre symbol modulo $p$. Let $m_{p}$ be the cardinality of a maximal such set $S$. The authors estimate the size of $m_{p}$.
\end{abstract}

1. Introduction. Let $p \equiv 1(\bmod 4)$ be a prime. A residue difference set modulo $p$ is a set of integers $\left\{a_{1}, \ldots, a_{k}\right\}$, with $1 \leqslant a_{i} \leqslant p-1$, such that

(i) $\left(\frac{a_{i}}{p}\right)=+1,1 \leqslant i \leqslant k$,

(ii) $\left(\frac{a_{i}-a_{j}}{p}\right)=+1,1 \leqslant i, j \leqslant k, i \neq j$,

where $\left(\frac{n}{p}\right)$ is the Legendre symbol modulo $p$. The maximal cardinality of a residue difference set modulo $p$ is denoted by $m_{p}$. The problem of estimating $m_{p}$ was posed at the West Coast Number Theory Conference in La Jolla, California in December 1976. We obtain the following estimates.

THEOREM. (i) $m_{p}>\frac{1}{2} \log p$ for all $p$,

(ii) $m_{p}<p^{1 / 2} \log p$ for all $p$,

(iii) $m_{p}<(1+\varepsilon) p^{1 / 2} \log p / 4 \log 2$ for all $p>C$, where $C \equiv C(\varepsilon)$ is a constant depending only on $\varepsilon$.

Any residue difference set can be transformed into a set containing 1 (by multiplication by any $\left.a_{i}^{-1}(\bmod p)\right)$, so we need only consider residue difference sets of the form

$$
S=\left\{a_{1}, a_{2}, \ldots, a_{k}\right\},
$$

where $1=a_{1}<a_{2}<\cdots<a_{k}$. Let $N_{p}(k)$ be the number of such sets. The value of $N_{p}(2)$ is exactly $(p-5) / 4$; we shall, in proving the theorem, obtain a lower bound for $N_{p}(k)$ for $k \geqslant 3$.

The proof of the theorem requires the following lemma, which we state here and prove in $\$ 3$.

Lemma. For any integer $k \geqslant 1$, let $a_{0}, a_{1}, \ldots, a_{k-1}$ be $k$ integers such that

Received by the editors March 14, 1977.

AMS (MOS) subject classifications (1970). Primary 10A15; Secondary 10G05.

' Research supported under National Research Council of Canada Grant No. A-7233. 
$a_{0}=0, a_{1}=1,1<a_{i}<p(i=2,3, \ldots, k-1), a_{i} \neq a_{j}$ for $i \neq j$. Set

$$
S\left(a_{0}, \ldots, a_{k-1}\right)=\sum_{\substack{x=0 \\ x \neq a_{0}, \ldots, a_{k-1}}}^{p-1}\left\{\prod_{j=0}^{k-1}\left(1+\left(\frac{x-a_{j}}{p}\right)\right)\right\} .
$$

Then $\left|S\left(a_{0}, \ldots, a_{k-1}\right)-p\right| \leqslant p^{1 / 2}\left\{(k-2) 2^{k-1}+1\right\}+k 2^{k-1}$, and if $p \geqslant$ $k^{2}$ the expression on the right-hand side of this inequality is at most $p^{1 / 2} k 2^{k-1}$.

Use will also be made of the following simple and easily-proved inequality: if $b_{1}, \ldots, b_{n}$ are $n(\geqslant 1)$ numbers such that $p \geqslant b_{1} \geqslant b_{2} \geqslant \cdots \geqslant b_{n}>0$ then

$$
\left(p-b_{1}\right) \cdots\left(p-b_{n}\right) \geqslant p^{n}-p^{n-1}\left(b_{1}+\cdots+b_{n}\right)
$$

2. Proof of the theorem. As $m_{5}=1, m_{13}=m_{17}=2, m_{29}=m_{37}=3, m_{41}=$ $m_{53}=4$, part (i) of the theorem is easily verified for $p \leqslant 53$. Thus we can assume $p \geqslant 61$, so that $\frac{1}{2} \log p>2$. In order to complete the proof we must show that $N_{p}(k)>0$ for $2 \leqslant k \leqslant \frac{1}{2} \log p$. To do this, we use the following expression for $N_{p}(k)$ :

$$
\begin{aligned}
& N_{p}(k)=\frac{1}{2^{(k-1)(k+2) / 2}} \sum_{\substack{a_{2}, \ldots, a_{k} \\
1<a_{2}<\cdots<a_{k}<p}}^{\sum_{p}}\left\{1+\left(\frac{a_{2}}{p}\right)\right\} \cdots\left\{1+\left(\frac{a_{k}}{p}\right)\right\} \\
& \cdot\left\{1+\left(\frac{a_{2}-1}{p}\right)\right\} \cdots\left\{1+\left(\frac{a_{k}-1}{p}\right)\right\} \\
& \prod_{2 \leqslant j<i<k}\left\{1+\left(\frac{a_{i}-a_{j}}{p}\right)\right\} \\
& =\frac{1}{2^{(k-1)(k+2) / 2}(k-1) !} \sum_{\substack{1<a_{2}<p \\
a_{i} \neq a_{j}, i \neq j}} \cdots \sum_{1<a_{k}<p}\left\{1+\left(\frac{a_{2}}{p}\right)\right\} \cdots \\
& \left\{1+\left(\frac{a_{k}}{p}\right)\right\} \\
& \cdot\left\{1+\left(\frac{a_{2}-1}{p}\right)\right\} \cdots\left\{1+\left(\frac{a_{k}-1}{p}\right)\right\} \\
& \prod_{2<j<i<k}\left\{1+\left(\frac{a_{i}-a_{j}}{p}\right)\right\} \\
& =\frac{1}{2^{(k-1)(k-2) / 2}(k-1) !} \sum_{1<a_{2}<p}\left\{1+\left(\frac{a_{2}}{p}\right)\right\}\left\{1+\left(\frac{a_{2}-1}{p}\right)\right\} \\
& \cdots \sum_{\substack{1<a_{k-1}<p \\
a_{k-1} \neq a_{2}, \ldots, a_{k-2}}}\left\{1+\left(\frac{a_{k-1}}{p}\right)\right\}\left\{1+\left(\frac{a_{k-1}-1}{p}\right)\right\} \\
& \cdot \prod_{j=2}^{k-2}\left\{1+\left(\frac{a_{k-1}-a_{j}}{p}\right)\right\} S\left(a_{0}, \ldots, a_{k-1}\right) .
\end{aligned}
$$


Since $p>\left(\frac{1}{2} \log p\right)^{2}$ (for all $p$ ) and as all the summands in the above expression for $N_{p}(k)$ are nonnegative, we can apply the lemma successively to obtain

$$
N_{p}(k) \geqslant \frac{1}{2^{(k-1)(k+2) / 2}(k-1) !}\left(p-2 \cdot 2 p^{\frac{1}{2}}\right) \cdots\left(p-k \cdot 2^{k-1} p^{\frac{1}{2}}\right) .
$$

Since for all integers $k \geqslant 2$ we have $\log (k-1)+k \log 2<k$, and as $k$ $\leqslant \frac{1}{2} \log p$, we obtain

$$
p^{1 / 2}>(k-1) 2^{k}>k 2^{k-1}
$$

Applying (1.1) we obtain

$$
\begin{aligned}
N_{p}(k) & \geqslant \frac{1}{2^{(k-1)(k+2) / 2}(k-1) !}\left\{p^{k-1}-p^{k-3 / 2}\left(2 \cdot 2+\cdots+k \cdot 2^{k-1}\right)\right\} \\
& =\frac{1}{2^{(k-1)(k+2) / 2}(k-1) !}\left\{p^{k-1}-(k-1) 2^{k} p^{k-3 / 2}\right\},
\end{aligned}
$$

and $N_{p}(k)>0$ follows from (2.1). Thus $m_{p}>\frac{1}{2} \log p$ for all primes $p$.

We now turn to the proofs of parts (ii) and (iii) of the theorem. The set of possible values of $a_{2}$ so that $\left\{1, a_{2}\right\}$ is a residue difference set modulo $p$ is

$$
A_{2}=\left\{b \mid\left(\frac{b}{p}\right)=\left(\frac{b-1}{p}\right)=+1\right\} \text {. }
$$

Fixing a value of $a_{2} \in A_{2}$, the set of possible values of $a_{3}$ so that $\left\{1, a_{2}, a_{3}\right\}$ is a residue difference set modulo $p$ is

$$
A_{3}=\left\{b \mid b \in A_{2},\left(\frac{b-a_{2}}{p}\right)=+1\right\} .
$$

Continuing in this way, one obtains for any residue difference set $S=\{1$, $\left.a_{2}, \ldots, a_{k-1}\right\}$, a set $A_{k}$ of possible values of $a_{k}$ so that $\left\{1, a_{2}, \ldots, a_{k}\right\}$ is a residue difference set. If $\alpha_{k}$ denotes the number of elements of $A_{k}$, then the residue difference set of maximal length that contains $S$ as a subset certainly has at most $k-1+\alpha_{k}$ elements, where

$$
\begin{array}{r}
\alpha_{k}=\frac{1}{2^{k}} \sum_{a_{k-1}<a_{k}<p}\left\{1+\left(\frac{a_{k}}{p}\right)\right\}\left\{1+\left(\frac{a_{k}-1}{p}\right)\right\}\left\{1+\left(\frac{a_{k}-a_{2}}{p}\right)\right\} \\
\cdots\left\{1+\left(\frac{a_{k}-a_{k-1}}{p}\right)\right\} \\
\leqslant \frac{1}{2^{k}} \sum_{\substack{a=0 \\
a \neq a_{0}, a_{1}, \ldots, a_{k-1}}}^{p-1} \prod_{i=0}^{k-1}\left\{1+\left(\frac{a-a_{i}}{p}\right)\right\}=\frac{1}{2^{k}} S\left(a_{0}, \ldots, a_{k-1}\right) .
\end{array}
$$

Thus, if $m_{p} \geqslant k-1$, there exists a set $S=\left\{1, a_{2}, \ldots, a_{k-1}\right\}$ which is a subset of a residue difference set of $m_{p}$ elements, and

$$
m_{p} \leqslant k-1+\frac{1}{2^{k}} S\left(a_{0}, \ldots, a_{k-1}\right) .
$$


Hence from the lemma we have

$$
\begin{aligned}
m_{p} & \leqslant k-1+\frac{1}{2^{k}}\left\{p+p^{1 / 2}\left((k-2) 2^{k-1}+1\right)+k 2^{k-1}\right\} \\
& \leqslant \frac{3 k}{2}-1+\frac{p}{2^{k}}+\frac{(k-1)}{2} p^{1 / 2} .
\end{aligned}
$$

If we now choose $k=1+[\log p / 2 \log 2]$, we see that $m_{p} \geqslant[\log p / 2 \log 2]$ implies

$$
m_{p} \leqslant \frac{3}{4 \log 2} \log p+\frac{1}{2}+p^{1 / 2}+\frac{p^{1 / 2} \log p}{4 \log 2} .
$$

Now for $p \geqslant 37$ we have

$$
\begin{aligned}
m_{p} & \leqslant\left(\frac{3}{4 \sqrt{37} \log 2}+\frac{1}{2 \sqrt{37} \log 37}+\frac{1}{\log 37}+\frac{1}{4 \log 2}\right) p^{1 / 2} \log p \\
& <(0.18+0.03+0.28+0.37) p^{1 / 2} \log p \\
& =0.86 p^{1 / 2} \log p \\
& <p^{1 / 2} \log p .
\end{aligned}
$$

As the inequality $m_{p}<p^{1 / 2} \log p$ is easy to check for $p=5,13,17$ and 29 , this completes the proof of (ii).

Part (iii) follows by choosing $p \geqslant C(\varepsilon)$ so that

$$
\frac{3}{4 \log 2} \log p+\frac{1}{2}+p^{1 / 2} \leqslant \varepsilon \frac{p^{1 / 2} \log p}{4 \log 2} .
$$

3. Proof of lemma. Let $f(x)=\left(x-c_{1}\right) \cdots\left(x-c_{t}\right)$, where the $c_{i}$ are $t$ $(\geqslant 1)$ integers which are incongruent modulo an odd prime $p$. Then the following estimate is a consequence of a deep result of $A$. Weil (see for example [1], [2]):

$$
\left|\sum_{x=0}^{p-1}\left(\frac{f(x)}{p}\right)\right| \leqslant(t-1) p^{1 / 2} .
$$

The term corresponding to the product of the l's in $S\left(a_{0}, \ldots, a_{k-1}\right)$ is

$$
\sum_{\substack{x=0 \\ x \neq a_{0}, \ldots, a_{k-1}}}^{p-1} 1=p-k .
$$

A typical term amongst the remaining $2^{k}-1$ terms is

$$
\sum_{\substack{x=0 \\ x \neq a_{0}, \ldots, a_{k-1}}}^{p-1}\left(\frac{\left(x-a_{i_{1}}\right) \cdots\left(x-a_{i_{r}}\right)}{p}\right)
$$

where $k \geqslant r \geqslant 1,0 \leqslant i_{1}<\cdots<i_{r} \leqslant k-1$. By (3.1) this sum is bounded in absolute value by $(r-1) p^{1 / 2}+k-r$. We thus have 


$$
\begin{aligned}
\mid S\left(a_{0}, \ldots,\right. & \left.a_{k-1}\right)-(p-k) \mid \leqslant \sum_{r=1}^{k}\left\{(r-1) p^{1 / 2}+(k-r)\right\}\left(\frac{k}{r}\right) \\
& =\left(p^{1 / 2}-1\right) \sum_{r=1}^{k} r\left(\begin{array}{l}
k \\
r
\end{array}\right)-\left(p^{1 / 2}-k\right) \sum_{r=1}^{k}\left(\begin{array}{l}
k \\
r
\end{array}\right) \\
& =\left(p^{1 / 2}-1\right) k 2^{k-1}-\left(p^{1 / 2}-k\right)\left(2^{k}-1\right) \\
& =p^{1 / 2}\left\{(k-2) 2^{k-1}+1\right\}+\left\{k 2^{k-1}-k\right\},
\end{aligned}
$$

so that

$$
\left|S\left(a_{0}, \ldots, a_{k-1}\right)-p\right| \leqslant p^{1 / 2}\left\{(k-2) 2^{k-1}+1\right\}+k 2^{k-1} .
$$

If $p \geqslant k^{2}$ then the right-hand side of the above is

$$
\begin{aligned}
& \leqslant p^{1 / 2}\left\{(k-2) 2^{k-1}+1+2^{k-1}\right\} \\
& \leqslant p^{1 / 2} k 2^{k-1} .
\end{aligned}
$$

4. Remarks. We note that the above arguments can be slightly refined to obtain marginal improvements in the constants appearing in the theorem. However, it appears to be a difficult problem to obtain the true order of magnitude of $m_{p}$. We have computed $N_{p}(k)$ and $m_{p}$ for all primes $p \leqslant 617$ and observed that for $p$ in the range $401 \leqslant p \leqslant 617, m_{p} / \log p$ varies between 1.27 and 1.72. One might expect, therefore, that $m_{p} \sim c \log p$ for some constant $c$ with $1 \leqslant c \leqslant 2$. However, our arguments, unless significantly modified, would not seem to yield a result of the type $m_{p} \geqslant \log p$.

The residue difference sets modulo $p$ form a tree with the nodes of the second level corresponding to the elements of $A_{2}$, the nodes of the third level corresponding to the elements of all sets $A_{3}$, etc. The computation of $N_{p}(k)$ was done by a depth-first search through this tree on the Xerox Data Systems Sigma 9 computer at Carleton University. As an indication of the number of nodes involved we note that for $p=617$ there were $1,374,659$ nodes.

\section{REFERENCES}

1. D. A. Burgess, The distribution of quadratic residues and non-residues, Mathematika 4 (1957), $106-112$.

2. On character sums and primitive roots, Proc. London Math. Soc. (3) 12 (1962), $179-192$.

Department of Mathematics, Carleton University, Ottawa (K1S 5B6), Ontario, Canada (Current address of K. S. Williams)

Current address (D. A. Buell): Department of Computer Science, Bowling Green State University, Bowling Green, Ohio 43403 